\title{
Role of Reference Levels in Nuclear Medicine: A Report of the SNMMI Dose Optimization Task Force
}

\author{
Adam M. Alessio ${ }^{1}$, Mary Beth Farrell ${ }^{2}$, and Frederic H. Fahey ${ }^{3}$ \\ ${ }^{I}$ Department of Radiology, University of Washington, Seattle, Washington; ${ }^{2}$ Intersocietal Accreditation Commission, Ellicott City, \\ Maryland; and ${ }^{3}$ Department of Radiology, Boston Children's Hospital, Harvard Medical School, Boston, Massachusetts
}

$\mathbf{T}$ he concept of reference levels has had a long history in diagnostic imaging (1). In general, reference levels provide guidance regarding appropriate or conventional levels of radiation dose to be delivered to patients. In Europe, the concept has origins in the 1950s with x-ray examination surveys in the United Kingdom (2). In the United States, the use of reference levels began with "Nationwide Evaluation of X-Ray Trends," a 1974-1981 survey (3). Since then, concepts have been more formalized in the international community by publications from the International Commission on Radiological Protection (ICRP) (4-7) and in the United States by the National Council on Radiation Protection and Measurements (NCRP) (8). With increased awareness of the potential risks from ionizing radiation, numerous recent publications have been addressing and suggesting reference levels. These publications offer some differing definitions of reference levels and raise some philosophic questions about their origin, purpose, and appropriate use (9-14). In this article, we discuss some considerations for application of reference levels to nuclear medicine in response to the ICRP Committee 3 recommendations encouraging "authorized bodies to set diagnostic reference levels that best meet their specific needs and that are consistent for the regional, national, or local area to which they apply" (7).

We will focus on two widely accepted concepts regarding reference levels: diagnostic reference levels (DRLs) and achievable doses (ADs). DRLs are set at the 75th percentile of radiation doses for examinations (5) and provide an investigational level to help identify unusually high doses. ADs are set at the median (50th percentile) of radiation doses for examinations $(8)$ and are intended to identify common practice. When DRLs and ADs are established, the distribution of radiation doses is typically determined by a survey of clinical sites based on protocol reviews, actual patient data, or phantom experiments. Here, we will refer to both DRLs and ADs as reference levels. This terminology is not to be confused with the term reference level as used by some publications to describe interventional radiologic examinations, or diagnostic reference level as used to describe diagnostic examinations (8).

Along with a growing consensus on the definition of DRL and AD, there is also general agreement that these should not be interpreted as absolute measures of appropriate use of medical radiation $(5,9)$.

Received May 13, 2015; revision accepted Sep. 10, 2015.

For correspondence or reprints contact: Adam M. Alessio, University of Washington, Box 357987, 4000 15th Ave. NE, Seattle, WA 98195-7987.

E-mail: aalessio@uw.edu

Published online Sep. 24, 2015.

COPYRIGHT (C) 2015 by the Society of Nuclear Medicine and Molecular Imaging, Inc.

DOI: $10.2967 /$ jnumed.115.160861
Reference levels should be used only to supplement, not replace, professional judgment and do not provide a dividing line between good and bad medicine. They are not intended for regulatory or commercial purposes or to establish legal standards of care. Even with these agreed constraints on the role of reference levels in diagnostic imaging, several philosophic and practical questions remain open about them. Should all clinics follow the same national reference levels? Is there some leeway within which a clinic would be considered compliant with the reference levels? What is the appropriate source for setting reference levels? Are reference levels set solely for radiation protection, or should they also guide appropriate image quality? How can clinics use reference levels for protocol optimization and ultimately improved patient care?

\section{ROLE OF REFERENCE LEVELS IN IMAGING}

\section{Education}

In a broad sense, reference levels offer a tool to educate imaging clinics on best practices. National reference levels can be used to create more unified imaging practices nationwide. Local reference levels, potentially set by each clinic to meet unique needs, can be used to ensure appropriate, consistent practice for radiation safety and optimal image quality within individual clinics.

\section{Optimum Range}

Application of reference levels "promotes attainment of an optimum range of values for a specified medical imaging protocol" (7).

\section{Action Levels}

DRLs can be used as suggested action levels. If a patient did or will receive more radiation than the DRL, the facility should review that patient's dosing and determine whether an improvement is possible. Such an improvement can come in the form of different dosing, better equipment, or a protocol modification. In some instances, the site may determine that this level of exposure is, in fact, appropriate for that particular patient.

\section{Normal Levels}

ADs can be used to define normal practice to help ensure that most examinations are performed near the AD level.

It is widely agreed that reference levels are not intended to replace good judgment. Each clinic has a unique set of factors (scanner technology, time for examinations, patient population, physician preference) that can dictate radiation doses (and local reference levels) that deviate from published levels. As clearly stated in the practice parameter of the American College of Radiology and the American Association of Physicists in Medicine, "The specific purpose of the diagnostic reference level is to provide a benchmark for comparison, not to define a maximum or minimum dose limit" (9). 


\section{NATIONAL OR LOCAL LEVELS}

The ICRP defines DRL as a level set by professional advisory bodies or societies implying a national or regional value that applies to numerous imaging centers (5). Many European countries have published reference levels for a variety of diagnostic examinations $(1,11,15)$. In the United States, NCRP report 172 suggested recommended reference levels for many ionizing radiation examinations, ranging from dental radiographs to nuclear medicine procedures (8). In this context, the reference levels must be applicable to a wide range of clinics. Therefore, as Wall and Shrimpton suggest, the reference level "should not be set at an 'optimum' or 'minimum achievable' level but more at the borderline between acceptable and unacceptable practice" (1). Because of the need to set the level at the high end of acceptable, our view is that national reference levels serve primarily as guidance to ensure that excessive radiation doses are not delivered. Consequently, we assert that national reference levels have a limited role in promoting optimal practice-that is, sufficient image quality at the minimum dose.

In contrast, each clinic can set local reference levels based on local resources (imaging equipment, time for examinations, physician experience). Local reference levels can also evolve on the basis of practice changes (improvements in imaging equipment, changes in physician experience) and thus should be reviewed regularly.

National reference levels serve primarily as guidance to ensure that certain radiation doses are not exceeded, and local reference levels serve as a tool to optimize protocols. One could argue that DRLs are the radiation protection measure (message: do not exceed the DRL) and ADs are the normal practice/improvement measure (message: try to practice close to AD). When set by national and regional bodies, both these levels must still be fixed at the high end of acceptable practice, limiting the ability to refine protocols to be close to the "as low as reasonably achievable" level (16).

\section{RADIATION DOSE VERSUS PROTOCOL DESIGN}

Many publications agree that reference levels help "optimize patient radiation dose and image quality" (9) and, similarly, that they "represent an important tool to optimize image quality and the radiation dose delivered to patients" (8). DRLs are defined as upper thresholds for dose, and ADs are defined as middle targets for dose. Both imply that certain radiation doses should not be exceeded; this is primarily a radiation protection view. These levels do not provide insight into a case in which the radiation dose may be too low to achieve an image of sufficient diagnostic quality. They do not offer a lower threshold for dose, only an upper threshold.

With the image-quality limitation acknowledged, an upper-limit reference level can still be a useful tool. Its value is increased if the level is set close to optimal for a particular clinic, with optimal being defined as providing sufficient diagnostic quality at the minimum necessary radiation dose. This is another reason to promote local reference levels over national levels. A clinic with rationally selected reference levels that match its clinical resources can use these levels as tools for radiation protection (usually, practice below the local DRL) and for general image quality assurance (practice as close to local ADs as reasonable). The development of review tools and templates by national and international organizations to help clinics derive reference levels specific to their practice would be valuable.

\section{CONSIDERATIONS FOR NUCLEAR MEDICINE REFERENCE LEVELS}

We refer readers to NCRP 172 for an exhaustive list of recommended national reference levels in the United States for numerous modalities (8). The setting of reference levels should be based on an easily measured and standardized quantity. For nuclear medicine, all reference levels have been defined using activity. Administered activity provides a good measure for ensuring radiation protection. The radiation dose to the patient, although dependent on numerous factors (patient size, biokinetics), is linearly related to administered activity.

When it comes to image quality in nuclear medicine, administered activity does not tell the whole story. For x-ray-based imaging modalities, reference levels are based on accepted measures of dose indices from each modality. For example, in CT, reference levels are based on the absorbed dose to a standard phantom (13). These absorbed dose indices are directly proportional to image quality-specifically, to the photon density in the images. In other words, for x-ray modalities, the absorbed dose information is a strong predictor of image quality and therefore serves as a good metric for image quality assurance. In contrast, in nuclear medicine, the photon density in the image is directly proportional to administered activity but also to acquisition duration. In some respects, administered activity tells less than half the story about the quality of a study. Furthermore, unlike CT scanners and diagnostic radiography equipment, which have sensitivity variations on the order of $0 \%-40 \%$ between similar systems, there can be greater variations in sensitivity in nuclear medicine because of the different system geometries and collimators. For example, a dualhead camera will have a 2 -fold increase in sensitivity compared with a single-head camera. The wide range of nuclear medicine equipment means that the same activity administered to all patients at all clinics will not equate to equivalent quality in all images. This reality limits the role of administered activity reference levels in ensuring appropriate image quality in nuclear medicine practice.

We suggest using reference levels based on administered activity for the purpose of radiation protection guidance. To overcome part of the image-quality limitation, we also propose using a new quantity-administered activity duration product (ADP) - to help ensure that sufficient image quality is achieved for an examination. The ADP, in MBq-min, can easily be calculated for an examination by multiplying the administered activity (MBq) by the study duration (min). For SPECT imaging, considering that most systems are dual-head cameras, we will define this measure for the duration of a dual-head acquisition. If a single- or triple-head camera is used for the acquisition, the local value should be normalized accordingly, that is, $\left(\mathrm{ADP}_{\text {single-head }}=\right.$ $\mathrm{ADP} \times 2 ; \mathrm{ADP}_{\text {triple-head }}=\mathrm{ADP} \times 2 / 3$ ).

\section{ORIGIN OF NUCLEAR MEDICINE REFERENCE LEVELS}

The recommended doses for diagnostic nuclear medicine examinations have been based on the initial trials with each tracer. Typically, these doses were set by the initial investigators to achieve sufficient diagnostic quality and eventually led to the recommended doses on package inserts. Since then, there have been limited or no adjustments to most of the recommended doses. They often span a wide range, and clinics in the United States generally do not adhere to them.

The nuclear medicine community does not have sufficient data to determine common dosing and acquisition strategies at clinics 
TABLE 1

Average Dosing for ${ }^{99 m}$ Tc-MDP Bone SPECT from IAC Survey Data

\begin{tabular}{lcccc}
\hline \multicolumn{1}{c}{ Site type } & Sites $(n)$ & $\begin{array}{c}\text { Scans/year } \\
(n, \text { average })\end{array}$ & $\begin{array}{c}\text { Dosing } \\
(\text { range-based and fixed) }\end{array}$ & 99mTc-MDP dose \\
\hline Hospital & 111 & 759 & $61 \%$ & $905 \pm 112[710-1,236] \mathrm{MBq}, 24.5 \pm 3.0[19.2-33.4] \mathrm{mCi}$ \\
\hline Private & 50 & 220.2 & $53 \%$ & $973 \pm 125[738-1,215] \mathrm{MBq}, 26.3 \pm 3.4[20.0-32.9] \mathrm{mCi}$ \\
Free-standing & 36 & 490.6 & $68 \%$ & $918 \pm 107[740-1,180] \mathrm{MBq}, 24.8 \pm 2.9[20.0-31.9] \mathrm{mCi}$ \\
\hline Multispecialty & 27 & 278.1 & $38 \%$ & $953 \pm 98[740-1,206] \mathrm{MBq}, 25.7 \pm 2.6[20.0-32.6] \mathrm{mCi}$ \\
\hline Mobile & 1 & 78.3 & $100 \%$ & $1,315 \mathrm{MBq}, 35.5 \mathrm{mCi}$ \\
Total & 225 & 543.8 & $58 \%$ & $930 \pm 118[710-1,315] \mathrm{MBq}, 25.1 \pm 3.2[19.2-35.5] \mathrm{mCi}$
\end{tabular}

Doses are mean \pm SD followed by minimum to maximum in brackets.

in the United States. As stated on page 73 of NCRP 172, "Determining reference levels for commonly performed nuclear medicine studies is challenging due to limited available survey data..." (8). In the absence of sufficient survey data, the NCRP published some suggested adult reference levels based on a small survey of 9 academic centers. It is unlikely that sampling of these few sites is sufficient to provide administered activities indicative of the broader practice of nuclear medicine in the United States.

\section{NEW SURVEY DATA FOR ${ }^{99 M}$ TC-MDP AND ${ }^{18}$ F-FDG}

To better understand normal practice in the United States, we evaluated the administration schemes of clinics that submitted accreditation applications to the Intersocietal Accreditation Commission (IAC) between February 2008 and December 2012. We evaluated dosing for ${ }^{99 \mathrm{~m} T c-m e t h y l e n e ~ d i p h o s p h o n a t e ~}\left({ }^{99 \mathrm{~m}} \mathrm{Tc}-\right.$ MDP) bone SPECT and ${ }^{18}$ F-FDG whole-body PET scans. This study did not require institutional review board approval because it does not involve protected health information; the included data were fully anonymized according to standards set by privacy rule section 164.514(a)-(c) of the Health Insurance Portability and Accountability Act. The average administered activity for each facility was calculated from patient case reports. Sites were categorized on the basis of their type (hospital, private, mobile), region of the country, and reported dosing strategy (fixed, range, or weight-based).

Tables 1 and 2 summarize the dosing strategies for ${ }^{99 m} \mathrm{Tc}-$ MDP and ${ }^{18}$ F-FDG scans categorized by type of facility.
Figure 1 presents histograms of injected activity for all cases submitted to the IAC in accreditation materials. For ${ }^{99 \mathrm{~m}} \mathrm{Tc}-\mathrm{MDP}$ scans, 225 facilities were evaluated. For each facility, the average administered activity was calculated from the patient cases submitted for accreditation. Each facility submitted $1-4$ cases, with an average of $2.2 \pm 0.8$ cases contributing to the facility average. The average 99m Tc-MDP activity across facilities was $930 \pm 118 \mathrm{MBq}(25.1 \pm$ $3.2 \mathrm{mCi}$ ), with a dosing range of 710-1,315 MBq (19.2-35.5 mCi). In addition, $58 \%$ of facilities reported that they used a rangebased dosing strategy, whereas the remaining facilities reported using fixed dosing. Comparing average administered activity across 5 different clinic types (ranging from hospitals to mobile clinics), there were no significant or clinically relevant differences in dosing strategies. The single mobile clinic was noteworthy for using $40 \%$ more activity than other types of sites. Likewise, there were no significant differences between different regions of the country (no differences between Southeast, Southwest, Northeast, Northwest, or Midwest.

For ${ }^{18}$ F-FDG scans, 95 facilities were evaluated. Each facility submitted $1-5{ }^{18}$ F-FDG cases, with an average of $4.3 \pm 1.3$ cases submitted per facility. The average ${ }^{18} \mathrm{~F}$-FDG activity across facilities was $508 \pm 117 \mathrm{MBq}(13.7 \pm 3.2 \mathrm{mCi})$, with a range of $108-$ $875 \mathrm{MBq}$. For dosing strategies, $64 \%$ of clinics reported using a range of doses, $29 \%$ using fixed dosing, and $7 \%$ using weightbased dosing. Like ${ }^{99 m} \mathrm{Tc}-\mathrm{MDP}$, there was no relevant difference in average dosing across clinic types. Again, the 3 mobile clinics were noteworthy for using roughly $30 \%$ more activity than the

TABLE 2

Average Dosing for ${ }^{18}$ F-FDG Whole-Body PET from IAC Survey Data

\begin{tabular}{lcccc}
\hline \multicolumn{1}{c}{ Site type } & Sites $(n)$ & $\begin{array}{c}\text { Scans/year } \\
(n, \text { average })\end{array}$ & $\begin{array}{c}\text { Dosing } \\
\text { (range-based:fixed:weight-based) }\end{array}$ & 18F-FDG dose \\
\hline Hospital & 41 & $1,345.8$ & $25: 10: 5$ & $532 \pm 102[372-875] \mathrm{MBq}, 14.4 \pm 2.8[10.1-23.7] \mathrm{mCi}$ \\
\hline Private & 16 & 653 & $12: 3: 1$ & $519 \pm 73[360-620] \mathrm{MBq}, 14.0 \pm 2.0[9.7-16.8] \mathrm{mCi}$ \\
Free-standing & 27 & 1,036 & $14: 10: 0$ & $476 \pm 114[134-761] \mathrm{MBq}, 12.9 \pm 3.1[3.6-20.6] \mathrm{mCi}$ \\
\hline Multispecialty & 8 & 513.1 & $5: 2: 0$ & $421 \pm 204[108-622] \mathrm{MBq}, 11.4 \pm 5.5[2.9-16.8] \mathrm{mCi}$ \\
Mobile & 3 & $4,661.2$ & $2: 1: 0$ & $632 \pm 39[608-677] \mathrm{MBq}, 17.1 \pm 1.1[16.4-18.3] \mathrm{mCi}$ \\
Total & 95 & 1,274 & $58: 26: 6$ & $508 \pm 117[108-875] \mathrm{MBq}, 13.7 \pm 3.2[2.9-23.7] \mathrm{mCi}$
\end{tabular}

Doses are mean \pm SD followed by minimum to maximum in brackets. 

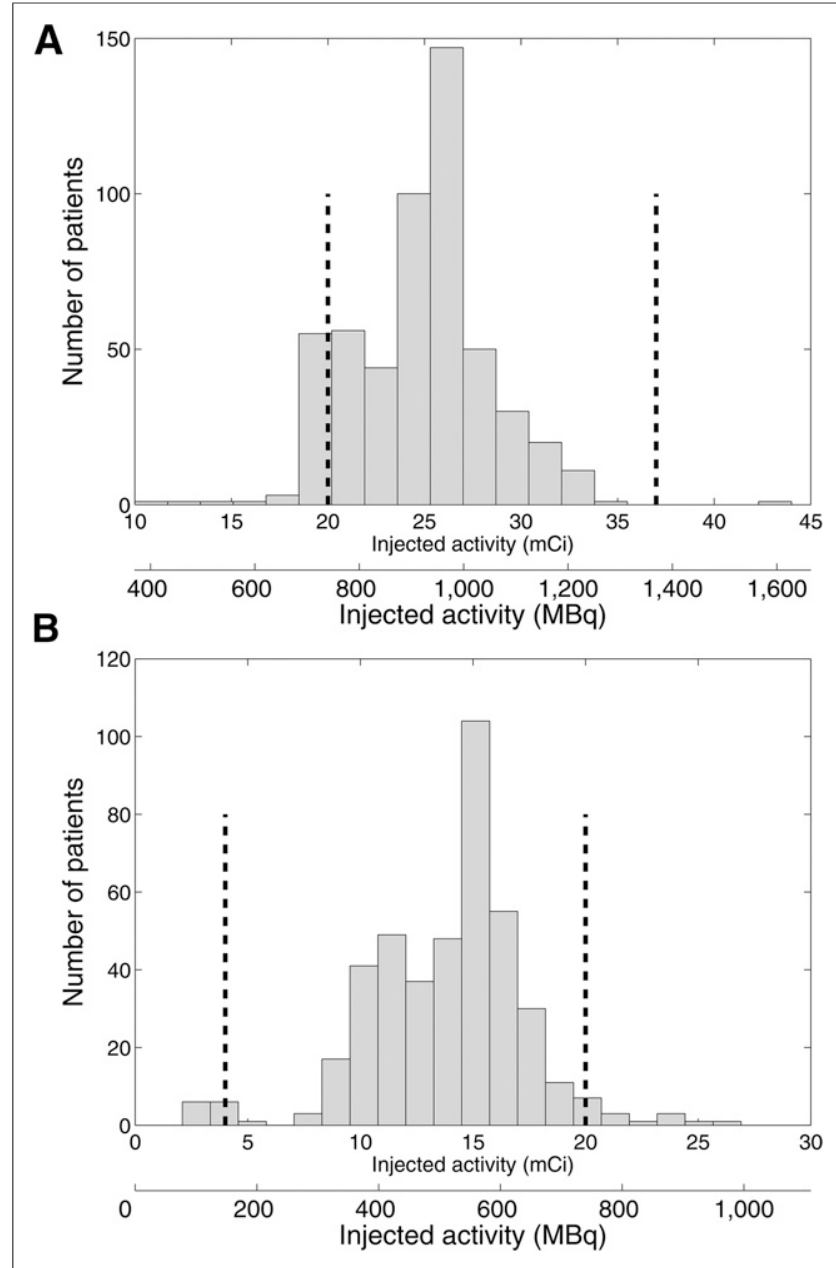

FIGURE 1. Histogram of injected activity for all 99mTc-MDP (A) and ${ }^{18} \mathrm{~F}-\mathrm{FDG}(\mathrm{B})$ cases submitted to IAC in accreditation materials. ${ }^{99 m T c-M D P}$ histogram represents 522 patient cases from 225 separate facility applications. ${ }^{18}$ F-FDG histogram represents 424 patient cases from 95 separate facility applications. Dashed lines mark lower and upper limits of recommended range from SNMMI guidelines.

other types of clinics. Likewise, there were no relevant differences between regions of the country.
From these data, we derived the DRLs and ADs from the 75th and 50th percentiles of the facility distributions of average dosing. Table 3 summarizes the Society of Nuclear Medicine and Molecular Imaging (SNMMI) recommended dosing ranges, the NCRP 172 reference levels, and the values for ${ }^{99 \mathrm{~m}} \mathrm{Tc}-\mathrm{MDP}$ and ${ }^{18} \mathrm{~F}-\mathrm{FDG}$ from our IAC survey. The ADs were similar between NCRP and our IAC survey. The DRLs that were suggested in the NCRP document were higher than the 75th percentile in the IAC survey. Our understanding is that the 9-site survey that contributed to the NCRP levels did not include a survey of actual patient dosings from which the 75th percentile dose levels could be extracted. Rather, this survey queried the minimum, maximum, mean, and median dosing based on prescribed practice. With this dataset, the NCRP suggested the DRL to be the 75th percentile of the maximum levels (this is not representative of the 75th percentile of all injected activities). This DRL level (75th percentile of maximum values) will most certainly be higher than the ICRP-defined DRL (75th percentile of all values) and helps explains the deviation of the DRLs from NCRP and from our IAC survey.

Table 3 also includes suggested ADP reference levels. For the ${ }^{99 m}$ Tc-MDP SPECT examinations, we used a total acquisition duration of 25 min times the IAC survey results to present AD and DRL levels for ADP. For ${ }^{18}$ F-FDG PET, we used an acquisition duration of 3 min per bed position for the ADP levels. These represent suggested national levels that could be refined by clinics to provide local levels.

\section{DISCUSSION}

Reference levels are primarily intended to offer benchmark values as a rough guideline for appropriate practice. With this goal in mind, one could argue that reference levels do not need to be exact but rather serve as general suggestions for appropriate, normal practice. We argue that the more general and rough the reference levels are, the less value they offer for determining appropriate practice. For example, if the $99 \mathrm{~m}$ Tc-MDP DRL is $1,185 \mathrm{MBq}$ (32 $\mathrm{mCi}$ ), as suggested by NCRP 172 , this number is sufficiently high that most clinics will never need to consider improvements, leading to little value to the field. In contrast, if the 99m Tc-MDP DRL is $999 \mathrm{MBq}(27 \mathrm{mCi})$, more clinics will have cause for reflection and potential improvements. Furthermore, if a clinic decides that local resources dictate a local ${ }^{99 \mathrm{~m}} \mathrm{Tc}-\mathrm{MDP}$ DRL of $1,050 \mathrm{MBq}(28 \mathrm{mCi})$, then the site has a tailored, rational value to ensure good practice for its patients.

TABLE 3

Recommended Adult Reference Levels for 99mTc-MDP Bone SPECT and ${ }^{18}$ F-FDG Whole-Body PET

\begin{tabular}{|c|c|c|c|c|c|c|c|}
\hline \multirow[b]{3}{*}{ Examination } & \multirow[b]{3}{*}{ SNMMI } & \multicolumn{4}{|c|}{ Radiation protection: injected activity } & & \\
\hline & & \multicolumn{2}{|c|}{ NCRP 172} & \multicolumn{2}{|c|}{ IAC survey } & \multicolumn{2}{|c|}{ Image quality: ADP* } \\
\hline & & $A D$ & DRL & $A D$ & DRL & $A D$ & DRL \\
\hline $\begin{array}{l}\text { 99mTc-MDP } \\
\text { bone SPECT }\end{array}$ & $\begin{array}{c}740-1,110 \mathrm{MBq} \\
(20-37 \mathrm{mCi})\end{array}$ & $\begin{array}{l}833 \mathrm{MBq} \\
(23 \mathrm{mCi})\end{array}$ & $\begin{array}{c}\text { 1,185 MBq } \\
\text { (32 mCi) }\end{array}$ & $\begin{array}{l}925 \mathrm{MBq} \\
(25 \mathrm{mCi})\end{array}$ & $\begin{array}{l}999 \mathrm{MBq} \\
(27 \mathrm{mCi})\end{array}$ & $\begin{array}{r}23,100 \mathrm{MBq} \cdot \min \\
(625 \mathrm{mCi} \cdot \mathrm{min})\end{array}$ & $\begin{array}{c}25,000 \mathrm{MBq} \cdot \min \\
(675 \mathrm{mCi} \cdot \mathrm{min})\end{array}$ \\
\hline $\begin{array}{l}{ }^{18} \mathrm{~F}-\mathrm{FDG} \\
\text { whole-body } \\
\text { PET }\end{array}$ & $\begin{array}{c}148-740 \mathrm{MBq} \\
\quad(4-20 \mathrm{mCi})\end{array}$ & $\begin{array}{l}555 \mathrm{MBq} \\
\quad(15 \mathrm{mCi})\end{array}$ & $\begin{array}{l}710 \mathrm{MBq} \\
\quad(19 \mathrm{mCi})\end{array}$ & $\begin{array}{l}518 \mathrm{MBq} \\
\quad(14 \mathrm{mCi})\end{array}$ & $\begin{array}{l}592 \mathrm{MBq} \\
\quad(16 \mathrm{mCi})\end{array}$ & $\begin{array}{c}\text { 1,554 MBq.min } \\
(42 \mathrm{mCi} \cdot \mathrm{min})\end{array}$ & $\begin{array}{c}\text { 1,776 MBq.min } \\
\text { (48 } \mathrm{mCi} \cdot \mathrm{min})\end{array}$ \\
\hline
\end{tabular}

${ }^{\star 99 m}$ Tc-MDP bone SPECT values assume use of dual-head system with 25-min study duration. ${ }^{18} \mathrm{~F}-\mathrm{FDG}$ whole-body PET values assume acquisition with 3 min per bed position. 
For national reference levels intended to reflect normal practice, there are currently limited data on nuclear medicine practice in the United States. We present survey data from 2 common examinations, ${ }^{18}$ F-FDG whole-body PET and ${ }^{99 m}$ Tc-MDP bone SPECT. This type of analysis needs to be expanded to more types of examinations to better understand practice patterns around the United States and provide nationwide reference levels.

Finally, reference levels can also be used for pediatric imaging. The NCRP document reported results from a 2007 survey of 13 pediatric hospitals by Treves et al. (17). Caution should be used in basing reference levels on these older survey data. The intention of that survey was not to suggest appropriate dosing but to highlight the variability among pediatric nuclear medicine clinics. That survey led to the North American Consensus Guidelines, which were recently updated $(18,19)$. A follow-up to the 2007 survey performed after adoption of the North American Consensus Guidelines has recently been published (20). Furthermore, given the wide range of pediatric patient sizes and appropriate dosing levels, a single reference level for pediatric nuclear medicine is arguably of little value. Pediatric patient dosing (and procedures for ensuring safe dosing) should be tailored for the patient; the North American and European guidelines recommend weight-based dosing schemes $(19,21)$.

\section{IMPLEMENTATION OF NUCLEAR MEDICINE REFERENCE LEVELS}

We recommend that clinics adopt local nuclear medicine reference levels for administered activity and ADP as a tool for radiation protection, for protocol improvement, and to ensure best practices. One suggested approach is initially to set local DRLs and ADs on the basis of national standards (such as the values presented in Table 3) and then to refine these local levels to meet the demands and evolution of the local clinical practice. Local levels should be reviewed and modified if necessary during structured protocol reviews to determine whether improvements are needed or possible. For example, if image quality is consistently higher or lower than deemed necessary, doses and acquisition durations should be modified accordingly. Likewise, if clinical resources change (more/less time for each imaging session, improved equipment, different physician preference/experience), reference levels should be modified.

\section{CONCLUSION}

The main points of this work are that national reference levels can provide guidance and an educational tool for comparison with regional practice. Local reference levels, based on the unique resources and conditions of the particular clinic, should be used if the intention is to inform local protocol selection. For nuclear medicine, reference levels based on administered activity offer a tool for radiation protection but have a limited role in ensuring appropriate image quality. Reference levels based on both radiation dose and acquisition duration can help ensure radiation protection and appropriate image quality.

\section{DISCLOSURE}

The costs of publication of this article were defrayed in part by the payment of page charges. Therefore, and solely to indicate this fact, this article is hereby marked "advertisement" in accordance with 18 USC section 1734. No potential conflict of interest relevant to this article was reported.

\section{ACKNOWLEDGMENTS}

The contributing members from the SNMMI Dose Optimization Task Force are Adam M. Alessio, Frederic H. Fahey, Jonathan Frey, Michael G. Stabin, and S. Ted Treves

\section{REFERENCES}

1. Wall BF, Shrimpton PC. The historical development of reference doses in diagnostic radiology. Radiat Prot Dosimetry. 1998;80:15-19.

2. Adrian E. Committee on Radiological Hazards to Patients. Br J Radiol. 1957;30:285

3. Burkhart R. Nationwide Evaluation of X-Ray Trends (NEXT): Eight Years of Data (1974-1981). Springfield, VA: National Technical Information Service; 1984.

4. Recommendations of the International Commission on Radiological Protection. Oxford, U.K.: Pergamon Press; 1991. ICRP publication 60.

5. Recommendations of the International Commission on Radiological Protection. Oxford, U.K: Pergamon Press; 1996. ICRP publication 73.

6. Avoidance of Radiation Injuries from Interventional Procedures. Oxford, U.K.: Pergamon Press; 2000. ICRP publication 85.

7. Diagnostic Reference Levels in Medical Imaging: Review and Additional Advice. Oxford, U.K.: Pergamon Press; 2002. ICRP Supporting Guidance 2.

8. Reference Levels and Achievable Doses in Medical and Dental Imaging: Recommendations for the United States. Bethesda, MD: National Council on Radiation Protection and Measurements; 2012. NCRP report 172.

9. ACR-AAPM practice parameter for diagnostic reference levels and achievable doses in medical x-ray imaging. In: American College of Radiology Practice Guidelines and Technical Standards. Reston, VA: American College of Radiology; 2014:1-9.

10. Etard C, Celier D, Roch P, Aubert B. National survey of patient doses from whole-body FDG PET-CT examinations in France in 2011. Radiat Prot Dosimetry. 2012;152:334-338.

11. Roch P, Aubert B. French diagnostic reference levels in diagnostic radiology, computed tomography and nuclear medicine: 2004-2008 review. Radiat Prot Dosimetry. 2013;154:52-75.

12. Goske MJ, Strauss KJ, Coombs LP, et al. Diagnostic reference ranges for pediatric abdominal CT. Radiology. 2013;268:208-218.

13. McCollough C, Branham T, Herlihy V, et al. Diagnostic reference levels from the ACR CT accreditation program. J Am Coll Radiol. 2011;8:795-803.

14. Mettler FAJ, Bhargavan M, Faulkner K, et al. Radiologic and nuclear medicine studies in the United States and worldwide: frequency, radiation dose, and comparison with other radiation sources-1950-2007. Radiology. 2009;253: 520-531.

15. Rehani MM, Tsapaki V. Impact of the International Atomic Energy Agency (IAEA) actions on radiation protection of patients in many countries. Radiat Prot Dosimetry. 2011;147:34-37.

16. Slovis TL. The ALARA concept in pediatric CT: myth or reality? Radiology. 2002;223:5-6.

17. Treves ST, Davis RT, Fahey FH. Administered radiopharmaceutical doses in children: a survey of 13 pediatric hospitals in North America. J Nucl Med. 2008;49:1024-1027.

18. Gelfand MJ, Parisi MT, Treves ST. Pediatric radiopharmaceutical administered doses: 2010 North American consensus guidelines. J Nucl Med. 2011;52:318322 .

19. Lassmann M, Treves ST. Pediatric Radiopharmaceutical Administration: harmonization of the 2007 EANM Paediatric Dosage Card (version 1.5.2008) and the 2010 North American Consensus guideline [comment]. Eur J Nucl Med Mol Imaging. 2014;41:1636.

20. Fahey FH, Ziniel SI, Manion D, Treves ST. Effects of Image Gently and the North American guidelines: administered activities in children at 13 North American pediatric hospitals. J Nucl Med. 2015;56:962-967.

21. Pediatric injected activity tool (version 1.02b). SNMMI website. http://www. snmmi.org/ClinicalPractice/PediatricTool.aspx. Published April 16, 2014. Accessed October 1, 2015. 